Chimia $46(1992) 6-9$

(C) Schweiz. Chemiker-Verband; ISSN 0009-4293

\title{
Chemiker HTL: heute und morgen
}

\author{
Heiner G. Bührer*
}

Chemikerinnen und Chemiker HTL sind in der industriellen Praxis geschätzte Kaderleute, doch ist ihre Ausbildung und die Bedeutung ihres Berufstandes leider zu wenig bekannt. Der folgende Artikel will deshalb-als Einstieg zu den nachfolgenden Porträts der einzelnen Schulen - einige Fragen beantworten: Wer wird Chemiker HTL? Welche Schulen bieten dieses Studium an? Wie sind die Berufsaussichten in einem Europa des Umbruchs? Welches sind die Weiterbildungsmöglichkeiten?

\section{Wege zum Chemiker}

Chemiker (Bezeichnungen wie Chemiker, Dozent, Student sollen in diesem Artikel für beide Geschlechter gelten) werden in der Schweiz in der sog. Tertiärstufe auf zwei grundsätzlich verschiedenen Wegen ausgebildet, nämlich

- anschliessend an die Matura mit Studium an einer Universität oder Technischen Hochschule oder

- anschliessend an eine Berufslehre (mit berufsbegleitender Schule) mit Studium an einer Ingenieurschule IS (= Höhere Technische Lehranstalt, HTL):

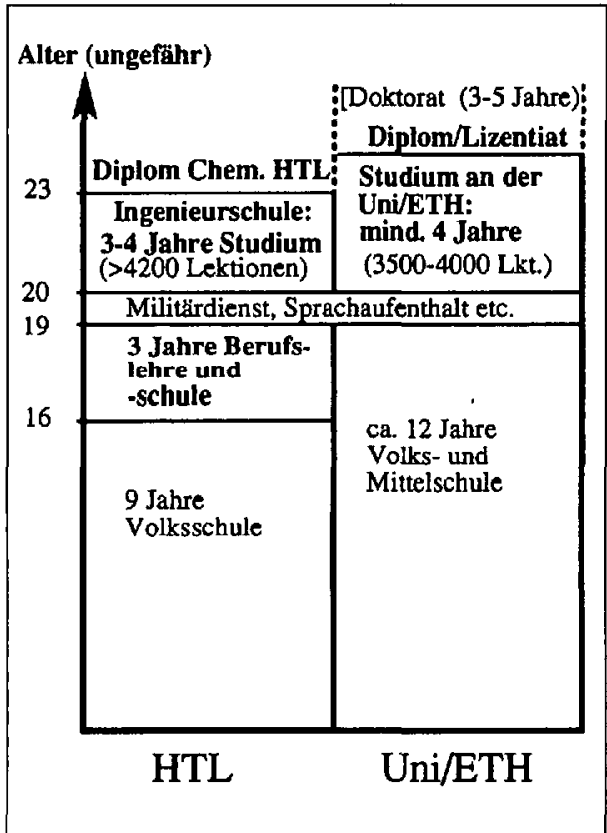

Fig. 1. Duales System zur Chemiker-Ausbildung in der Schuciz

\footnotetext{
*Korrespondenz: Prof. H.G. Bührer Technikum Winterthur Ingenieurschule, TWI Postfach 805 CH-8401 Winterthur
}

Dieses duale System ist in seiner heutigen Form für IS-Absolventen wenig durchlässig (s. aber Kap. 4). Es lässt sich am ehesten mit dem deutschen Ausbildungssystem, bei dem allerdings die 'Ingenieurschule' in den siebziger Jahren durch die 'Fachhochschule' abgelöst wurde, vergleichen. Andere Länder, z.B. Grossbritannien, besitzen zwar ebenfalls eine duale Ausbildungsstruktur, nämlich Universitäten und sog. Polytechnics. Letztere bauen aber nicht auf der Berufslehre auf, sondern besitzen oft eine 'Sandwich-Struktur' mit zwischengeschalteter Industriepraxis [1].

Wie setzen sich die Chemikerdiplome zahlenmässig zusammen? In Fig. 2 sind die Abschlusszahlen von 1977-89 zusammengetragen:

Demnach werden etwa die Hälfte aller Diplome bzw. Lizentiate in Chemie an Hochschulchemiker, 14\% an HochschulChemieingenieure und $36 \%$, d.h. ein gutes

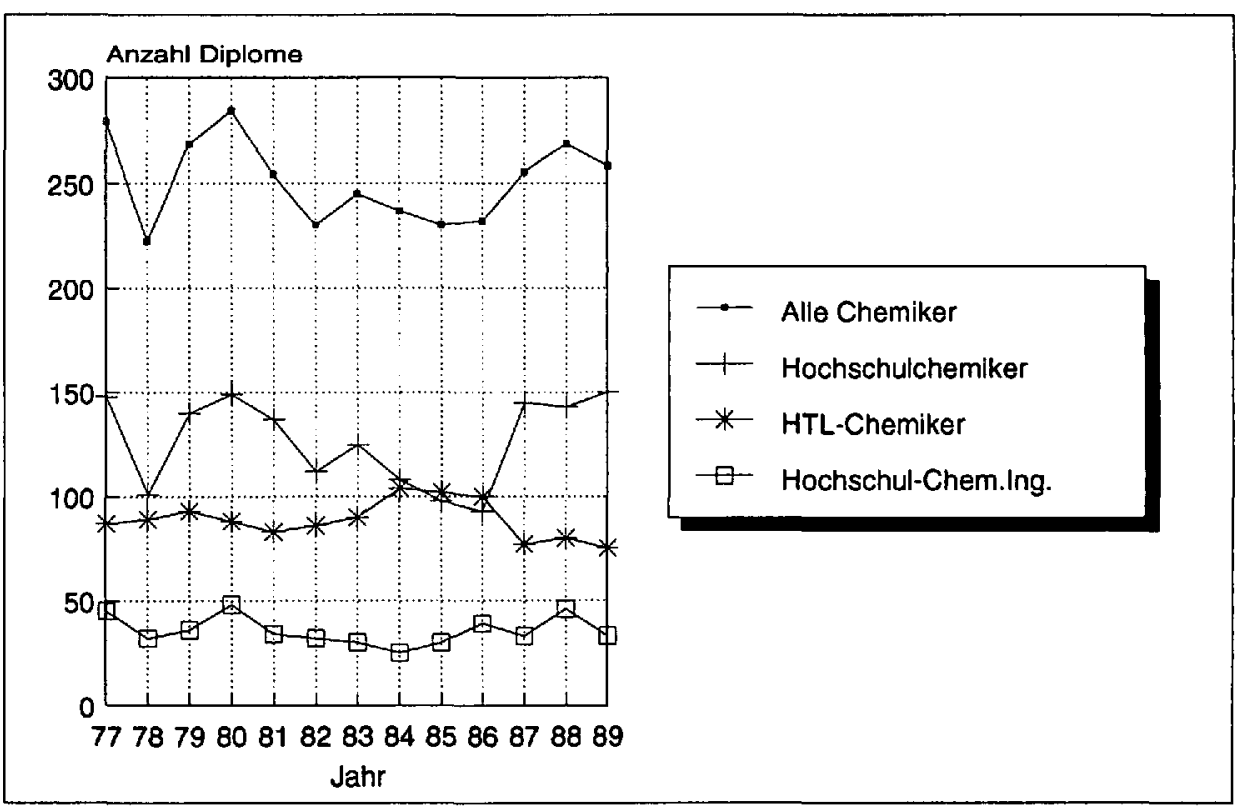

Fig. 2. Diplome in Chemie an Hoch- und Ingenieurschulen der Schweiz von 1977-89
Drittel, an HTL-Chemiker verliehen. Diese Zahlen kontrastieren zur Gesamtmenge der Ingenieure: Über zwei Drittel aller Schweizer Ingenieure besitzen ein HTLDiplom! Aus Fig. 2 folgt eine zweite wichtige Aussage: Die Zahlen für die verschiedenen Diplomtypen sind über längere Zeiträume betrachtet bemerkenswert konstant und stagnieren seit den siebziger Jahren! Dies widerspricht dem generell zu beobachtenden Trend zu anspruchsvoller Ausbildung: In den letzten 10 Jahren hat die Studentenzahl der HTL Winterthur um $22 \%$, jene der Universität Zürich gar um $45 \%$ zugenommen. Demgegenüber blieb die Zahl der Phil.II-Studierenden in Zürich konstant. Dass diese Stagnation bei den Chemikerzahlen nicht auf ein mangelhaftes Stellenangebot zurückzuführen ist, belegt allein schon die Tatsache, dass heute in der Schweizer Grosschemie etwa jede zweite Chemikerstelle mit einem Ausländer besetzt werden muss.

Wie Fig. 1 zeigt, schliesst die HTLAusbildung an eine Berufslehre an (Maturanden beginnen nur in Ausnahmefällen ein HTL-Studium). Sie leidet damit unter dem Umstand, dass der Anteil der Mittelschüler an der 19 jährigen Gesamtbevölkerung in den letzten Jahren stetig zugenommen hat. Heute beträgt er rund $13 \%$ (schweizerisches Mittel; Kt. Genf 27\%, Basel-Stadt 21\%, Zürich 15\%, Bern und Graubünden je $9 \%$ ) und liegt damit zwar in Europa immer noch tief, aber doch weit höher als vor 10 oder 20 Jahren.

Damit stellt sich die Frage, wie die weitere Entwicklung aussehen könnte. Das mit Abstand grösste Nachwuchspotential stellt die Laborantenlehre dar (Chemikanten entschliessen sich nur selten für ein Studium). Die Aufteilung in die einzelnen Richtungen zeigt Fig. 3. 
Aus dieser Darstellung geht klar hervor, dass nur Chemie- und Biologielaboranten relevante Kontingente an Chemiestudierenden stellen können. Eine Prognose wird möglich, wenn die Entwicklung der neuen Lehrverträge betrachtet wird.

Die Zahl der Chemielaboranten ist also rückläufig, und die Zahl der Biologielaboranten stagniert. Biolaborantinnen übertreffen ihre männlichen Kollegen zahlenmässig im Verhältnis 2:1. Dies ist sicher mit ein Grund dafür, dass Biolaboranten an den Chemie-Abteilungen nur spärlich vertreten sind (daneben richtet sich die heutige Ausbildung bewusst an den Chemielaboranten, worüber in späteren Artikeln noch mehr berichtet wird). Da die Dauer zwischen Abschluss des Lehrvertrags und Chemikerdiplom bei rund $7 \mathrm{Jah}$ ren liegt (vgl. Fig. I), ist ab etwa 1993 eine Abnahme der Diplomzahlen anzunehmen, wenn nicht zusätzliche Nachwuchsquellen erschlossen werden können. Solche wären sicher bei den Frauen vorhanden, liegt doch ihr Anteil an den Chemiestudierenden immer noch zu tief (IS Winterthur ca. $15 \%$, zum Vergleich: Universität Zürich $20 \%$ )

\section{Die schweizerischen Ingenieurschu- len mit Chemie-Abteilung}

Schweizer Ingenieurschulen mit Chemie-Abteilung sind an folgenden Orten zu finden: Burgdorf (gegründet 1892), Chur (Abend-HTL, nimmt nur jedes zweite Jahr Studenten auf), Fribourg (erste Diplome 1977), Genève (Génie Chimique), Muttenz (erste Diplome 1974), Sion (erste Diplome 1991) und Winterthur (älteste Abteilung, gegründet 1875). Die Gesamt-

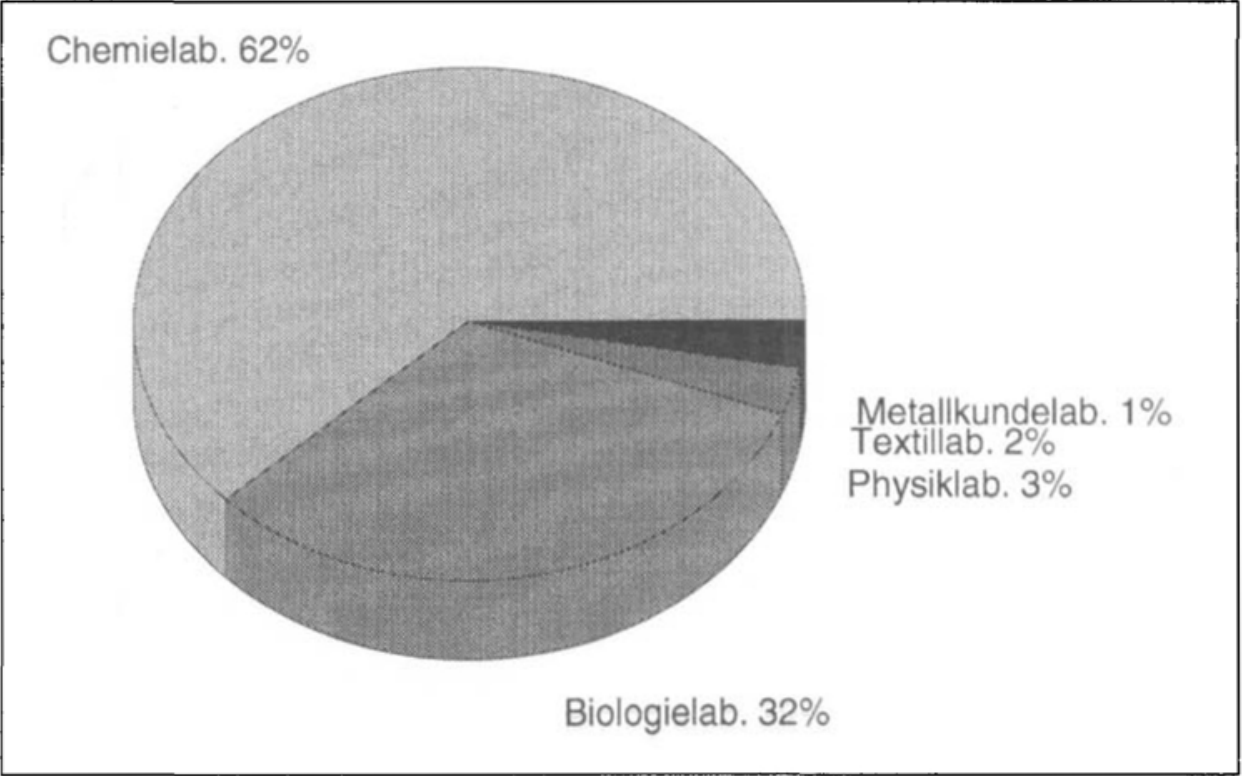

Fig. 3. Prozentuale Aufteilung der neuen Lehrverträge in Laborantenherufen (1990)

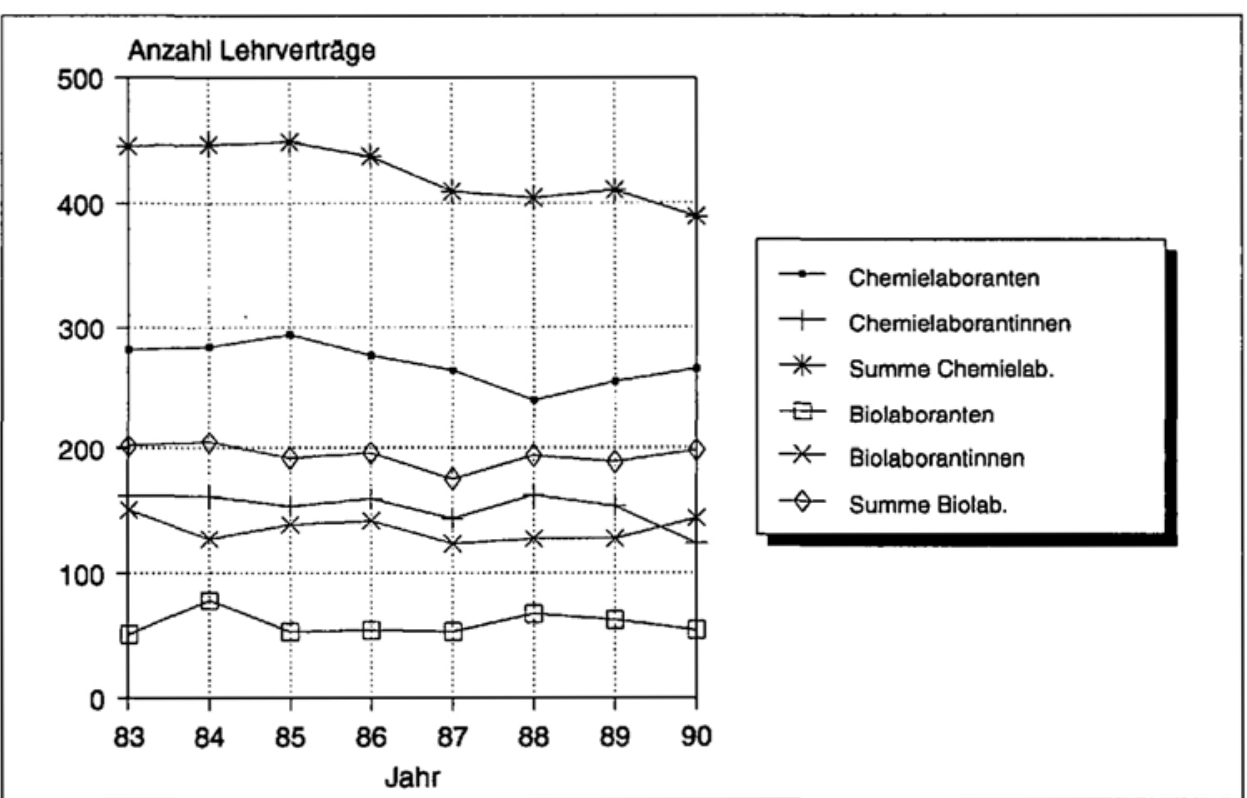

Fig. 4.Zahlenmässige Entwicklung bei den neuen Lehrverträgen für Chemie-und Biologielaborunten $(1983-90)$

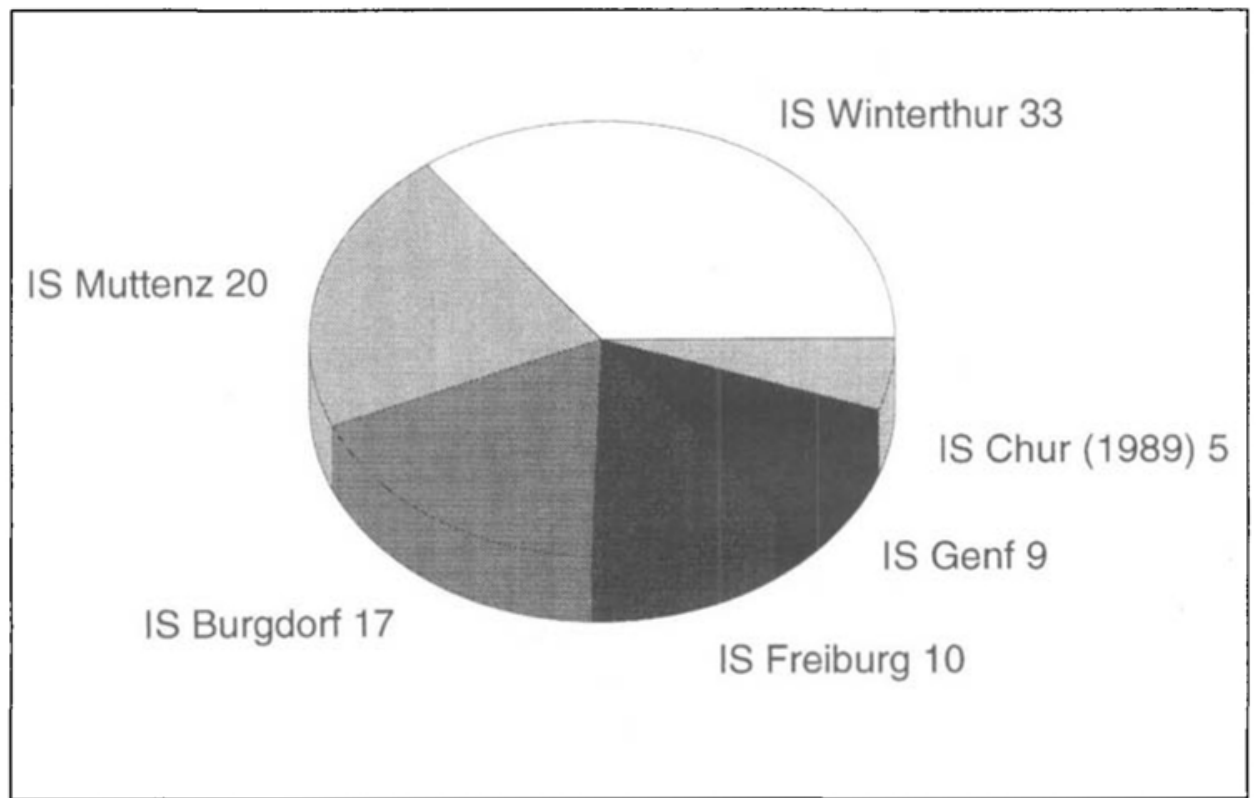

Fig. 5. Diplome in Chemie an den Ingenieurschulen der Schweiz (1990) zahl der Diplome betrug in den letzten 20 Jahren etwa 80 bis $100 / J a h r$, während im gleichen Zeitraum die Zahl der Schulen von 4 auf 7 wuchs. Die Verteilung der Diplome auf die einzelnen Ingenieurschulen zeigt Fig. 5 .

An der Ingenieurschule Winterthur befindet sich die mit Abstand grösste Chemie-Abteilung mit zwei Klassenzügen. Einige Ingenieurschulen halten zahlenmässig durchaus einen Vergleich mit den Universitäten aus: 1990 wurden an den Schweizer Universitäten folgende Lizentiate und Diplome in Chemie erteilt: Basel 20 , Bern 31 , Fribourg 8 , Genève 13, Lausanne 6, Neuchâtel 10, Zürich (Universität) 14 und ETH-Zürich 62, dazu kamen 20 bzw. 12 Diplome in Chemieingenieurwesen an den Eidgenössische Technischen Hochschulen ETH in Lausanne bzw. Zürich. 
Eine Chemie-Abteilung sollte eine kritische Grösse aus Kostengründen (Nutzung der im Vergleich zu andern Studienrichtungen teuren Infrastruktur), aber auch aus personellen Gründen (eine grössere Dozentenzahl ermöglicht eher eine fachliche Vertiefung auf einzelnen Gebieten) nicht unterschreiten. Zur Illustration einige Zahlen: Ein Studienplatz an der IS Winterthur kostete 1989 pro Student brutto Fr. 26 000.-, an der Universität Zürich mit dem hohen Anteil an 'billigen' Phil.IStudenten Fr. 27 000.-. Der Unterricht in relativ kleinen Klassen macht eine der Stärken der HTL-Ausbildung aus: die intensive Betreuung durch den Dozenten. An der IS Winterthur kommen auf einen Professor etwa 10 Studierende, an der ETH-Zürich aber 39 und an der Universität Zürich gar über 60 (alle Fakultäten) bzw. 30 Studierende (nur Phil.II). Allerdings ist diese intensive Betreuung - auch im Praktikumsunterricht - auch eine Folge des an Ingenieurschulen im Vergleich zu Universitäten knapp dotierten Mittelbaus (Assistenten).

Mit dem Stichwort 'Klassenbetrieb' ist ein erster Unterschied zwischen HTLund Hochschul-Ausbildung angesprochen. Ein zweiter Unterschied ergibt sich aus den verschiedenen Schwerpunkten bei der Primär- und Sekundärausbildung: Während der Maturand in mindestens 12 Jahren ein breites Allgemeinwissen mit geringer fachlicher Vertiefung erarbeitet, dominiert beim Laboranten nach etwa der gleichen Zeit die fachlich-praktische Komponente. Dieser Ausrichtung wird die Ingenieurschule gerecht: Ziel ist der Chemiker HTL als Entwickler, Optimierer und Realisator. Entsprechend ist das Studium breit angelegt und umfasst synthetische, analytische und - dies im Gegensatz zum Chemie-Studium an der Universität-auch

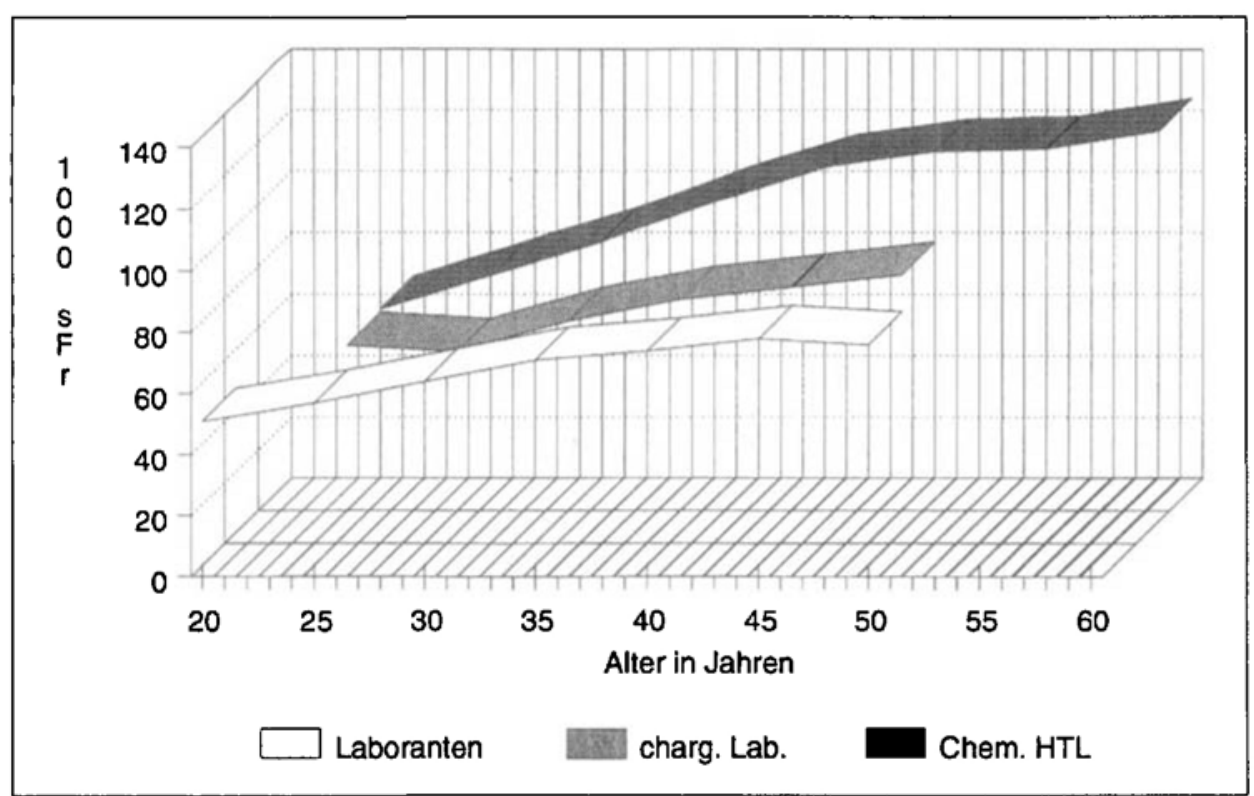

Fig. 6. Saläre in der Chemie (1990/91, Quellen SLZ [2] und SVCT [3]) ingenieurchemische Fächer. Allerdings lässt diese generalistische Ausbildung nur selten Spezialisierung und theoretische Tiefe zu. Dies hängt einerseits mit der kurzen Dauer der Diplomarbeiten (einige Wochen), andererseits aber mit der Stundenbelastung der Dozenten (typisch über 20 Lektionen) zusammen, die eine Mitarbeit an grösseren Forschungs- und Entwicklungsprojekten sehrerschwert. Trotzdem können einem jungen HTL-Chemiker in den meisten Tätigkeiten bereits nach kurzer Einarbeitungszeit Projekte zur selbständigen Bearbeitung übergeben werden. Auch sind ihm die Arbeit im Team und das betriebliche Umfeld aufgrund seiner Berufslehre nicht neu.

Eine leidige, aber schier unerschöpfliche Diskussion ist an der Titelfrage entbrannt. Während der ETH-Absolvent mit bestandener Diplomprüfung den Titel 'dipl. Chemiker ETH' trägt, blieb das 'dipl.' dem HTL-Chemiker trotz seines Diplomattests bisher verwehrt. Die neusten Entwicklungen in der Schweiz in Richtung EWR und EG lassen allerdings vermuten, dass ein Titel wie 'dipl. Chemiker HTL', 'dipl. Chemiker FH' oder auch einfach 'dipl. Chemiker' bald legal verwendet werden kann.

\section{Berufsaussichten}

Die Motive für einen Laboranten, ein Chemie-Studium an einer HTL zu beginnen, liegen vor allem auf vier Ebenen:

- fachliche Kompetenz,

- Arbeitsqualität,

- Salärentwicklung und

- Karrieremöglichkeiten.

Während die ersten beiden Ebenen schwer quantifizierbar sind, kann leicht gezeigt werden, dass das drei- bis vierjähBundesbehörden in Zusammenarbeit mit den betroffenen Schulen dieses Problem zügig anpacken und lösen werden.

\section{Weiterstudium und Weiterbildung}

Hier soll als erstes über das Weiterstudium an einer schweizerischen oder ausländischen Hochschule berichtet werden. Am besten bekannt ist die seit 1974 bestehende Übertrittsregelung HTL-ETHZ für sehr gut ausgewiesene Absolventen. Diese können nach einem einjährigen, gesamtschweizerisch an der IS Winterthur stattfindenden Übertrittskurs zu einer ausserordentlichen Aufnahmeprüfung ins 5. Semester derChemie-Abteilung derETHZ zugelassen werden. Der Zeitbedarf für das zusätzliche Diplom beträgt 3 Jahre. Aller- 
dings beenden nur wenige Chemiker so ihr Studium; die meisten beginnen anschliessend eine Doktorarbeit. Pro Jahr wählen einige wenige besonders begabte und motivierte Chemiker HTL diese Route.

Ein Weiterstudium ist prinzipiell auch an ausländischen Hochschulen möglich. Wegen der Bedeutung der englischen Sprache für die Berufswelt des Chemikers werden angelsächsische Universitäten bevorzugt. Ausschlaggebend sind hier persönliche Kontakte zwischen den Dozenten, da der Eintrag im Diplomausweis 'equivalent to a Bachelor-of-Science degree' allein noch keine Zulassung garantiert. Sehr gute Kontakte bestehen z.B. zwischen der University of Calgary, Kanada, und der Ingenieurschule Winterthur. Etwa 20 Ehemalige der IS Winterthur erwarben dort einen 'Master'- oder 'Ph.D.'-Grad (4-5 Jahre Zusatzstudium). Seit 1990 existiert auch ein Studentenaustausch für Praktikumsplätze in der Industrie ('Co-operative Education') [6].

Im Bestreben, die zunehmende Isolation unserer Ingenieurschulen in Europa zu durchbrechen, wurden auch Kontakte zu ähnlichen Schulen in Deutschland, Holland und England geknüpft (vgl. den Artikel über das NDS in Biotechnologie an der IS Winterthur). So konnte 1990 erstmals ein Diplomand des Autors seine Diplomarbeit an der Hogeschool Heerlen, Holland, abschliessen und kam damit in den Besitz eines zweiten, EG-konformen Diploms. Diese Aktivitäten werden mit zunehmenden Mobilitätswünschen der Studenten und Dozenten und weitergehender Internationalisierung von Wissenschaft und Technik in Zukunft an Bedeutung gewinnen.

Für die fachliche Weiterbildung des Chemikers bestehen eine Reihe von Angeboten: Betriebswirtschaftslehre, Biotechnologie, Energie, Informatik, Kunststofftechnik, Umweltschutz, Werkstofftechnik etc. (ein Teil dieser Fächer, wie etwa Umweltschutz oder Betriebswirtschaftslehre, treten bereits heute in HTLLehrplänen auf, können aber mit dem bestehenden Stundenangebot unmöglich in gebührender Tiefe behandelt werden). Es handelt sich dabei entweder um berufsbegleitende Nachdiplomkurse (meist Freitag/Samstag) oder um Vollzeitstudien (meist einjährig). In jedem Fall ist der zeitliche und finanzielle Aufwand beträchtlich.

Immer mehr wird auch von Arbeitgeberseite her anerkannt, dass es nicht nur Möglichkeiten, sondern eine Pflicht zur Weiterbildung gibt. Für die Ingenieurschulen eröffnet sich hier ein Potential, das auch weiteren Interessenten zugute kommen kann.

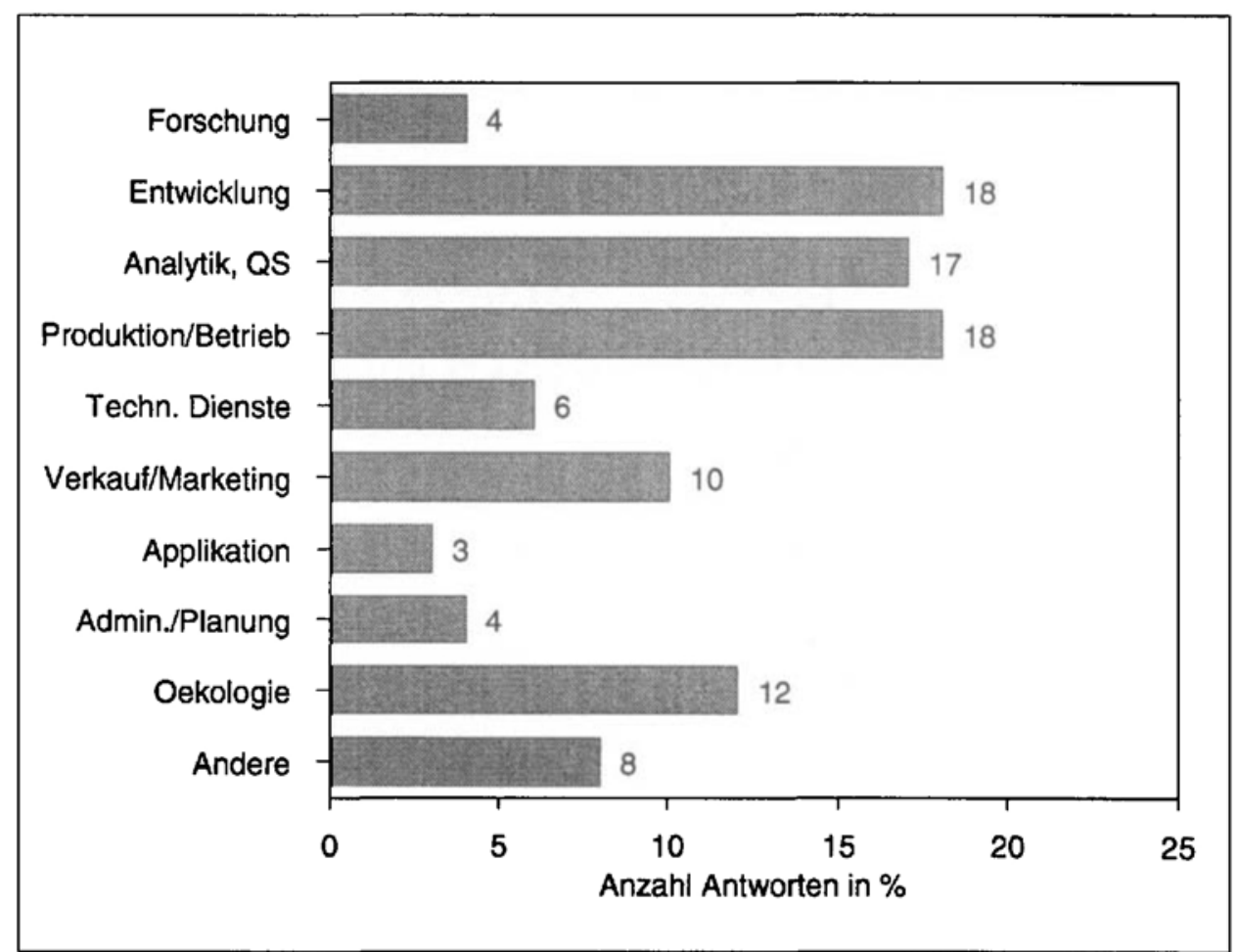

Fig. 7. Prozentuale Verteilung der Tätigkeiten von HTL-Chemikern gemäss SVCT-Umfrage 1991

\section{Offene Fragen}

Der Autor hofft, dass es ihm gelungen ist, die Probleme, aber auch die Chancen des Chemikers HTL aufzuzeigen. Auch die besten Ausbildungsstätten (dies betrifft gleichermassen Volksschulen, Mittelschulen, Ingenieurschulen und Universitäten) sind heute der Kritik und dem Vergleich mit dem Ausland ausgesetzt. Ein Ausruhen auf früher erworbenen Lorbeeren ist fahrlässig. Es ist deshalb nützlich, sich einigen kritischen Fragen zu stellen und sie zu beantworten:

- Ist eine Alternative zum dualen Ausbildungssystem denkbar und wie müsste sie aussehen?

- Ist unsere föderalistische Ausbildungsstruktur noch zeitgemäss?

- Müsste für eine Vergrösserung des Nachwuchspotentials an Chemikern HTL eine Steigerung der Attraktivität des Laborantenberufs ins Auge gefasst werden? Wird es längerfristig den Chemielaborantenberuf überhaupt noch geben?

- Wie könnte die Attraktivität des HTLStudiums für Biolaboranten gesteigert werden?

- Kann das HTL-Studium für praxisorientierte Maturanden zu einer echten Alternative zum Hochschulstudium werden?

- Stellt die - in einem späteren Artikel vorgestellte - Studienreform an der IS Winterthur die richtige Antwort auf die steigenden Ansprüche an die Chemikerausbildung dar?
- Ist die strikte Zuteilung: Universität = Lehre und Forschung, HTL = nur Lehre, heute noch sinnvoll?

- Genügen die Bestrebungen für eine europäische und internationale Anerkennung der HTL-Ausbildung?

- Sind die heutigen Bedingungen für ein Weiterstudium nach dem HTL-Diplom attraktiv?

- Soll das bestehende Angebot an Weiterbildungsmöglichkeiten ausgebaut werden?

- Könnten nicht-vor allem in der GrossChemie - viele Stellen statt mit einem Hochschulchemiker ohne Qualitätsverlust mit einen HTL-Chemiker besetzt werden?

Ihre Antworten auf diese Fragen, aber auch Reaktionen zu allen andern Artikeln dieses Heftes würden die Autoren freuen und sehr interessieren.

[1] K. Stephan, Chem.-Ing.-Tech. 1990, 62, 599.

[2] SLZ 1991, 48, 152

[3] N. Di Menna, SVCT-Salärumfrage 1991, Schweiz. Vereinigung dipl. Chem. HTL, Postfach 46, 4007 Basel.

[4] H. G. Bührer, Chimia 1989, 43, 23.

[5] J. U. Schlegel, Die EG als bildungspolitische Herausforderung, NZZ 1989, 273, 89.

[6] H. G. Bührer, Weiterbildungsmöglichkeiten für Winterthurer Absolventen, TECHinfo Technikum Winterthur Ingenieurschule, März 1990, 34. 